June 2021

revised October 2021

\title{
Midisuperspace foam and the cosmological constant
}

\author{
S. CARLIH* \\ Department of Physics \\ University of California \\ Davis, CA 95616 \\ USA
}

\begin{abstract}
Wheeler's conjectured "spacetime foam" - large quantum fluctuations of spacetime at the Planck scale - could have important implications for quantum gravity, perhaps even explaining why the cosmological constant seems so small. Here I explore this problem in a midisuperspace model consisting of metrics with local spherical symmetry. Classically, an infinite class of "foamy" initial data can be constructed, in which cancellations between expanding and contracting regions lead to a small average expansion even if $\Lambda$ is large. Quantum mechanically, the model admits corresponding stationary states, for which the probability current is also nearly zero. These states appear to describe a self-reproducing spacetime foam with very small average expansion, effectively hiding the cosmological constant.
\end{abstract}

*email: carlip@physics.ucdavis.edu 


\section{Spacetime foam and the cosmological constant}

More than 65 years ago, Wheeler suggested that quantum uncertainties in the metric should be of order one at the Planck scale, leading to large fluctuations in geometry and (perhaps) topology, which he called "spacetime foam" [1]. While this idea has continued to generate interest, it has been notoriously hard to investigate quantitatively. My goal here is to develop a model simple enough to allow calculations, but still complex enough to describe a large assortment of foam-like quantum states.

More specifically, one aim is to test a proposal that spacetime foam might help solve the cosmological constant problem. In [2], I suggested that our Universe might in fact have a large cosmological constant $\Lambda$, whose effects are "hidden" in Planck scale fluctuations. I showed that for a large class of initial data, the expansion and shear-the observational signature of a cosmological constant - average to zero over small regions even if $\Lambda$ is large. A slightly oversimplified picture is that a cosmological constant can cause either expansion or contraction; using a construction of Chrusciel, Isenberg, and Pollack [3,4], one can sew together Planck-scale regions with random initial data in a way that naturally leads to large cancellations.

A key question is whether such cancellations persist during evolution. This is fundamentally a matter for quantum gravity, and a full treatment lies beyond our current capabilities. There is, however, a partial step: we can explore mini- and midisuperspace models * in which many of the degrees of freedom are frozen out. Such models have been used to study a range of issues in quantum gravity, and while the results are rarely conclusive, they can be strongly suggestive.

Here I will focus on a midisuperspace consisting of geometries with local spherical symmetry. When combined with the "dust time" of Brown and Kuchař [8-10], the Wheeler-DeWitt equation takes a Schrödinger-like form, and can be solved by WKB methods. We shall see that there are stationary states in which a foamlike structure and small average expansion are preserved in time. Moreover, while regularization ambiguities exist, "foamy" spacetimes seem to occur with high probabilities. In this setting, at least, a cosmological constant may indeed be hidden in spacetime foam.

\section{Local spherical symmetry}

The setting for locally spherically symmetric midisuperspace has been studied extensively by Morrow-Jones, Witt, and Schleich [5 7]. At first sight, this symmetry requirement may seem too strong: it is widely believed that spherical symmetry with $\Lambda>0$ leads inevitably to Schwarzschild-de Sitter space. But while Birkhoff's theorem implies that a spherically symmetric vacuum spacetime must be locally isometric to some region of Schwarzschild-de Sitter space, local patches can be sewn together to form a spacetime that looks drastically different [7]. In particular, we will be able to construct explicit initial data containing both expanding and contracting regions.

Here, for simplicity, I will assume a positive cosmological constant, and specialize to the spatial topology $S^{1} \times S^{2}$. Recall that to construct a manifold with this topology, we start with

${ }^{*}$ Conventionally a minisuperspace keeps only finitely many degrees of freedom, while a midisuperspace has an infinite, although still restricted, set. 
a solid three-ball; cut out a ball from its center to form a manifold $[0,1] \times S^{2}$; and identify the boundaries $\{0\} \times S^{2}$ and $\{1\} \times S^{2}$. To build "foamy" spacetimes, we will take this construction a step further, splitting space into an onion-like sequence of concentric shells, each with its own geometry.

Following [5], let us start with the initial value formalism, with geometric data consisting of a spatial metric $q_{i j}$ and its conjugate momentum $\pi^{i}{ }_{j}$. These cannot be chosen arbitrarily, but must solve the momentum and Hamiltonian constraints

$$
\begin{aligned}
& \mathscr{P}_{j}={ }^{(3)} \nabla_{i} \pi^{i}{ }_{j}=0 \\
& \mathscr{H}=\frac{2 \kappa^{2}}{\sqrt{q}}\left(\pi^{i}{ }_{j} \pi^{j}{ }_{i}-\frac{1}{2} \pi^{2}\right)-\frac{1}{2 \kappa^{2}} \sqrt{q}\left({ }^{(3)} R-2 \Lambda\right)=0 .
\end{aligned}
$$

(I use the conventions of [11]; in particular, $\kappa^{2}=8 \pi G$.) On a hypersurface of constant time, a general locally spherically symmetric metric may be written in the form

$$
d s^{2}=h^{2} d \psi^{2}+f^{2}\left(d \theta^{2}+\sin ^{2} \theta d \varphi^{2}\right)
$$

where $h$ and $f$ are functions of $\psi$ and $t$. The spatial scalar curvature is

$$
{ }^{(3)} R=\frac{1}{f^{2}}\left[-\frac{4 f}{h}\left(\frac{f^{\prime}}{h}\right)^{\prime}-\frac{2 f^{\prime 2}}{h^{2}}+2\right],
$$

where a prime denotes a derivative with respect to $\psi$. Ref. [5] makes an added coordinate choice $h(\psi, t)=a(t), f(\psi, t)=a(t) \tilde{f}(\psi)$; this simplifies the classical solutions, but complicates the quantum treatment by gauge-fixing one of the diffeomorphism constraints.

The nonvanishing canonical momenta are

$$
\pi_{\theta}^{\theta}=\pi_{\varphi}^{\varphi}=\sin \theta Q, \quad \pi_{\psi}^{\psi}=\sin \theta P,
$$

where $P$ and $Q$ are functions of $\psi$ and $t$. (The $\sin \theta$ factors appear because the momenta are tensor densities). Geometrically, $P$ and $Q$ are extrinsic curvatures, fractional rates of expansion; explicitly,

$$
Q=\frac{1}{2 \kappa^{2}} h f^{2}\left(K_{\theta}^{\theta}+K_{\psi}^{\psi}\right), \quad P=\frac{1}{\kappa^{2}} h f^{2} K_{\theta}^{\theta} .
$$

The only nontrivial momentum constraint is

$$
\mathscr{P}=P^{\prime}-\frac{h^{\prime}}{h} P-\frac{2 f^{\prime}}{f} Q=0 \Rightarrow Q=\frac{f}{2 f^{\prime}}\left(P^{\prime}-\frac{h^{\prime}}{h} P\right),
$$

while the Hamiltonian constraint becomes, after a little algebra,

$$
\mathscr{H}=\frac{h}{f^{\prime}} \frac{d}{d \psi}\left[-\kappa^{2} \frac{P^{2}}{f h^{2}}+\frac{1}{\kappa^{2}}\left(\frac{f f^{\prime 2}}{h^{2}}-f\right)+\frac{\Lambda}{3 \kappa^{2}} f^{3}\right]=0 .
$$

This simple total derivative structure was first noted in [5]. 
Given a metric $(h, f)$, we can now solve (2.7) for $P$,

$$
P= \pm \frac{1}{\kappa^{2}}\left[f^{2} h^{2}\left(\frac{f^{\prime 2}}{h^{2}}-1\right)+\frac{\Lambda}{3} f^{4} h^{2}+\gamma f h^{2}\right]^{1 / 2}
$$

where $\gamma$ is an integration constant. As also noted in [5], this constant is a mass: if we look at a slice $P=0$ and gauge fix to $f=\psi$ (allowed locally, though not globally), we see that

$$
h^{2}=\left(1-\frac{\gamma}{\psi}-\frac{\Lambda}{3} \psi^{2}\right)^{-1},
$$

a piece of the usual Schwarzschild-de Sitter metric with $\gamma=2 G m$.

Note that the value of the integration constant $\gamma$ can have a drastic effect on the spacetime geometry [12,13]. Here I will assume that

$$
0<\gamma<\frac{2}{3 \sqrt{\Lambda}}
$$

For the complete Schwarzschild-de Sitter metric, this is the condition for the existence of two horizons, that is, for a black hole that is smaller than the cosmological horizon. As we will see shortly, it is also necessary for the existence of a Cauchy surface with vanishing extrinsic curvature, a requirement for attaching expanding and contracting regions to realize the "spacetime foam" of [2].

By standard existence theorems, the initial data $(h, f, P, Q)$ can now be evolved to form a maximal globally hyperbolic spacetime. As expected, the system has a time reversal symmetry: if the data $(h, f, P, Q)$ are admissible, so are $(h, f,-P,-Q)$. Since $P$ and $Q$ determine the expansion and shear, for any expanding solution there is a corresponding contracting one.

Of course, there is no guarantee that the resulting spacetimes are geodesically complete. On the contrary, regions of the initial surface can be isometric to regions of the interior of a Schwarzschild-de Sitter black hole, so one might generically expect singularities to form, as happens in the more general setting [14]. Evolution thus requires a quantum treatment.

\section{Sewing}

Ref. [2] focused on manifolds formed by "sewing" elementary pieces to build a model of spacetime foam. An almost identical procedure exists in the spherically symmetric setting [5]. Choose coordinates in which $h$ is constant in a small region around $\psi=\psi_{0}$. Pick two manifolds $M_{1}$ and $M_{2}$, with arbitrary functions $f_{1}(\psi)$ and $f_{2}(\psi)$. Let $u_{\epsilon}$ be a smoothed step function, interpolating between $u_{\epsilon}(\psi)=0$ when $\psi<-\epsilon$ and $u_{\epsilon}(\psi)=1$ when $\psi>\epsilon$.

Now define

$$
f(\psi)=\left[1-u_{\epsilon}\left(\psi-\psi_{0}\right)\right] f_{1}(\psi)+u_{\epsilon}\left(\psi-\psi_{0}\right) f_{2}(\psi) .
$$

This "sewn" $f$ looks like $f_{1}$ for $\psi \in\left(-\infty, \psi_{0}-\epsilon\right)$ and like $f_{2}$ for $\psi \in\left(\psi_{0}+\epsilon, \infty\right)$, with a smooth interpolation between. Since the momenta $P$ and $Q$ are determined locally from $f$ and $h$, the 
full initial data will sew together in the same way. Topologically, this process is a connected sum $M_{1} \# M_{2}$; geometrically, it is very close to the general construction of [3, 4].

This isn't yet quite good enough. The functions $f_{1}$ and $f_{2}$ each determine two sets of data, $\left(h, f_{1}, \pm P_{1}, \pm Q_{1}\right)$ and $\left(h, f_{2}, \pm P_{2}, \pm Q_{2}\right)$. We would like to sew any combination, for instance attaching an expanding region to a contracting region. But for $P$ and $Q$ to change sign, they must go through zero, a condition that is not automatic.

To allow such a sign change, we construct an extra "neck" $N$, an intermediate manifold in which $P$ and $Q$ go to zero on a central two-sphere. We can then sew $M_{1}$ and $M_{2}$ via this neck, $M_{1} \# N \# M_{2}$, with either choice of momenta on each side. We specify $N$, say with $\psi \in(-\delta, \delta)$, by demanding that

$$
f^{\prime}(0)=0, f^{\prime \prime}(0)>0, P(0)=Q(0)=0 .
$$

The condition $P(0)=0$ is easy: by (2.8), it simply requires that

$$
\frac{\Lambda}{3} f_{0}^{3}=f_{0}-\gamma
$$

where $f_{0}=f(0)$. Note, though, that (3.3) has real, positive roots only if the inequality (2.10) is satisfied [12. If the inequality is violated, $P$ can never go through zero, and it becomes impossible to join expanding and contracting regions, at least within the constraint of local spherical symmetry.

Setting $Q(0)=0$ is harder: eqn. (2.6) for $Q$ has a factor $f^{\prime}$ in the denominator, which goes to zero at the center of the neck. To cancel this term, we must demand that $P(\psi)$ be of order $\psi^{3}$ near $\psi=0$. A straightforward calculation yields the requirement

$$
f(\psi)=f_{0}+\left(1-\Lambda f_{0}^{2}\right) f_{0}\left(\frac{h^{2} \psi^{2}}{4 f_{0}^{2}}\right)\left(1-\frac{h^{2} \psi^{2}}{12 f_{0}^{2}}\right)+\mathcal{O}\left(\psi^{6}\right) \quad \text { with } 1-\Lambda f_{0}^{2}>0 .
$$

This restriction is not quite as stringent as it may seem: the $\mathcal{O}(1)$ and $\mathcal{O}\left(\psi^{2}\right)$ terms are already fixed by the demand that $Q$ remain finite at $f^{\prime}=0$.

By the constraint $(2.1 \mathrm{~b})$, the scalar curvature ${ }^{(3)} R$ is large at $\psi=0$, since the extrinsic curvature vanishes there. But the curvature falls off quickly away from the center of the neck:

$$
\begin{array}{r}
{ }^{(3)} R=2 \Lambda-\frac{8 \kappa^{4}}{h^{2} f^{4}}\left(P Q-\frac{1}{4} P^{2}\right)=2 \Lambda-\frac{2 \kappa^{4}}{3 h^{2}} \frac{1}{f_{0}^{3} f^{\prime \prime}(0)} P_{3}{ }^{2} \psi^{4}+\mathcal{O}\left(\psi^{5}\right) \\
\quad \text { where } P=\frac{1}{6} P_{3} \psi^{3}+\mathcal{O}\left(\psi^{4}\right) .
\end{array}
$$

We can now construct the promised "onion" initial data, with a mix of expanding and contracting layers. Choose a $K$-layer sequence $\psi_{0}<\psi_{1}<\cdots<\psi_{K}$, where $\psi_{K}$ will be identified with $\psi_{0}$ to create a closed $S^{1} \times S^{2}$. In each layer $\psi \in\left(\psi_{n}, \psi_{n+1}\right)$, choose functions $\left(h_{n}, f_{n}\right)$, and randomly pick the sign of the corresponding $\pm\left(P_{n}, Q_{n}\right)$. Sew successive layers along necks as described above. For a configuration with enough layers, the random choice of signs means that $P$ and $Q$ will typically average to near zero, even if $\Lambda$ is large. Thus, as in [2], we have hidden the initial cosmological constant, but now in a setting in which we can address the quantum theory much more concretely. 


\section{The problem of time}

To investigate the quantum evolution of this system, we must first confront a basic puzzle, the notorious "problem of time" [15, 16]. Quantum gravity is a relational theory, and time evolution can only be described relative to other degrees of freedom. To describe evolution, we must first choose a "clock."

In some expanding minisuperspace models [17, and perhaps more generally [18], one can use spatial volume as a clock. Here, though, our spacetimes have some regions that expand while others contract. One can often use "York time" [19], in which the trace of the extrinsic curvature - the local Hubble constant - serves as a clock. But here, again, we are interested in cases in which the extrinsic curvature doesn't even have a fixed sign at constant time. A more general choice of time, the mean curvature flow [20], fails for the same reason. In some cosmological spacetimes, "time since the big bang" can serve as a clock 21, but it is not obvious that the spacetimes considered here have a suitable initial big bang singularity.

An alternative, developed by Brown and Kuchař [8, 9], uses congruences of test particles, "dust," as a reference system This is not ideal - it would be preferable to describe the evolution purely in terms of a gravitational variable - but in the present setting I don't know how to define such a "clock." Here I will use a slightly simpler version of dust time, due to Husain and Pawłowski [10] (see also [22]), in which a space-filling congruence of irrotational dust is used to specify time evolution. The "cloud of clocks" is introduced with an action

$$
I_{\mathrm{dust}}=\frac{1}{2} \int d^{4} x \sqrt{-g} \rho\left(g^{a b} \partial_{a} T \partial_{b} T-1\right),
$$

where $\rho$ is a Lagrange multiplier. It is easy to see that the variation of $I_{\text {dust }}$ yields the usual stress-energy tensor for noninteracting irrotational matter. Carrying out a standard canonical decomposition of $I_{\text {grav }}+I_{\text {dust }}$, one finds constraints

$$
\begin{aligned}
& \mathscr{P}_{i}=\mathscr{P}_{i}^{\text {grav }}+\mathscr{P}_{i}^{\text {dust }}=0 \\
& \mathscr{H}=\mathscr{H}^{\text {grav }}+\mathscr{H}^{\text {dust }}=0,
\end{aligned}
$$

where $\mathscr{H}^{\text {grav }}$ and $\mathscr{P}_{i}^{\text {grav }}$ are given by $(2.1 \mathrm{a})-(2.1 \mathrm{~b})$ and

$$
\begin{aligned}
& \mathscr{P}_{i}^{\text {dust }}=-p_{T} \partial_{i} T, \\
& \mathscr{H}^{\text {dust }}=\left(p_{T}^{2}+q^{i j} \mathscr{P}_{i}^{\text {dust }} \mathscr{P}_{j}^{\text {dust }}\right)^{1 / 2},
\end{aligned}
$$

where $p_{T}$ is the momentum conjugate to $T$.

As shown in [8], one can use these constraints in a "many-fingered time" quantization, in which the wave function evolves as a functional of $T$. But one can also gauge fix time by choosing $T=t$. Physically, this amounts to using proper time along the dust worldlines as a time coordinate. With this choice, $\mathscr{P}_{i}^{\text {dust }}=0$, and the constraints reduce to

$$
\begin{aligned}
& \mathscr{P}_{i}^{\text {grav }}=0 \\
& p_{T}=\mathscr{H}^{\text {grav }} .
\end{aligned}
$$

\footnotetext{
${ }^{\dagger}$ Footnote 1 of 22 gives a history of this idea, which dates back to Einstein's "mollusc of reference."
} 
Eqn. (4.4b) has a slightly peculiar structure. The left-hand side is independent of position, since we have chosen a gauge in which $T$ depends only on time, but the right-hand side is certainly position-dependent. This is actually sensible: the zero mode of $\mathscr{H}^{\text {grav }}$ is now a true Hamiltonian, while the remaining position-dependent modes continue to act as constraints, much like the situation in unimodular gravity [23].

\section{Wheeler-DeWitt quantization}

To quantize this system a la Wheeler and DeWitt [25], we rewrite the momentum and Hamiltonian constraints as operators, with canonical momenta replaced by (functional) derivatives. To find the appropriate conjugates, we start with the symplectic current

$$
\omega=\int d^{3} x \pi^{i j} \delta q_{i j}=8 \pi \int d \psi\left(\frac{2}{f} Q \delta f+\frac{1}{h} P \delta h\right),
$$

from which can we read off

$$
P=\frac{i}{8 \pi} h \frac{\delta}{\delta h}, \quad Q=\frac{i}{16 \pi} f \frac{\delta}{\delta f}
$$

The spatial volume $\hat{V}$ and the mean curvature $\hat{K}$ (the trace of the extrinsic curvature) become

$$
\begin{aligned}
& \hat{V}=\int d^{3} x \sqrt{q}=4 \pi \int d \psi h f^{2} \\
& \hat{K}=-\frac{\kappa^{2}}{\sqrt{q}} \hat{\pi}=-\frac{i \kappa^{2}}{8 \pi} \frac{1}{h f^{2}}\left(h \frac{\delta}{\delta h}+f \frac{\delta}{\delta f}\right),
\end{aligned}
$$

and satisfy $[\hat{K}, \hat{V}]=-3 i \kappa^{2} / 2$.

The momentum constraint (2.6) now becomes

$$
\hat{\mathscr{P}}=\frac{i}{8 \pi}\left(h \partial_{\psi} \frac{\delta}{\delta h}-f^{\prime} \frac{\delta}{\delta f}\right) .
$$

Acting on $h$ and $f, \hat{\mathscr{P}}$ generates spatial diffeomorphisms, and invariant wave functions can be built from integrals of the form

$$
F[h, f]=\int d \psi h L\left[f, D f, D^{2} f, \ldots\right] \quad \text { with } D=\frac{1}{h} \frac{d}{d \psi} .
$$

The Hamiltonian constraint (2.7) is a bit more complicated, but can be reduced to the form

$$
\hat{\mathscr{H}}=\frac{h}{f^{\prime}} \frac{d}{d \psi}\left[\frac{\kappa^{2}}{64 \pi^{2}} \frac{1}{f} \frac{\delta^{2}}{\delta h^{2}}+\frac{1}{\kappa^{2}} f\left(\frac{f^{\prime 2}}{h^{2}}-1\right)+\frac{\Lambda}{3 \kappa^{2}} f^{3}\right],
$$

while the momentum $p_{T}$ of (4.4b) becomes

$$
p_{T}=i h f^{2} \frac{d}{d T}
$$


$\left(h f^{2}\right.$ is the volume measure $\left.\sqrt{q}\right)$. The Wheeler-DeWitt equation - the operator version of (4.4b), acting on a wave function $\Psi$-is thus

$$
i f^{2} f^{\prime} \frac{d \Psi}{d T}=\frac{d}{d \psi}\left[\frac{\kappa^{2}}{64 \pi^{2}} \frac{1}{f} \frac{\delta^{2}}{\delta h^{2}}+\frac{1}{\kappa^{2}} f\left(\frac{f^{\prime 2}}{h^{2}}-1\right)+\frac{\Lambda}{3 \kappa^{2}} f^{3}\right] \Psi .
$$

As in the classical case, (5.8) can be integrated. The derivative $d \Psi / d T$ is independent of position $-\Psi$ is a functional of $h$ and $f$, not a function of $\psi$ - so the left-hand side of (5.8) is a total derivative. Hence

$$
i \frac{d \Psi}{d T}=\left[\frac{3 \kappa^{2}}{64 \pi^{2}}\left(\frac{1}{f^{2}} \frac{\delta}{\delta h}\right)^{2}+\frac{3}{\kappa^{2}} \frac{1}{f^{2}}\left(\frac{f^{\prime 2}}{h^{2}}-1\right)+\frac{\Lambda}{\kappa^{2}}+\frac{3 \gamma}{\kappa^{2} f^{3}}\right] \Psi=\hat{H} \Psi,
$$

where $\gamma$ is again an integration constant. This is the fundamental equation we must solve to understand quantum midisuperspace.

\subsection{The wave function}

Eqn. (5.9) is a Schrödinger-type equation, and there is no difficulty in principle in interpreting $|\Psi|^{2}$ as a probability density on midisuperspace [15]. In particular, the time derivative

$$
\frac{d\left(\Psi^{*} \Psi\right)}{d T}=-\frac{3 i \kappa^{2}}{64 \pi^{2}} \frac{\delta}{\delta h}\left[\frac{1}{f^{4}}\left(\Psi^{*} \frac{\delta \Psi}{\delta h}-\Psi \frac{\delta \Psi^{*}}{\delta h}\right)\right]
$$

is a total (functional) derivative, so formally, the norm

$$
\int[d f][d h] \Psi^{*} \Psi
$$

is conserved, at least for suitable boundary conditions for midisuperspace.

There is a subtlety, though. The wave function $\Psi$ should really be defined on "reduced midisuperspace," the space of symmetric metrics modulo diffeomorphisms. To properly define a norm on this quotient space, one must gauge fix the inner product to avoid overcounting diffeomorphism-equivalent configurations [24]. This is a difficult task, since one must account for not only the spatial diffeomorphisms, but also the symmetries generated by the non-zero modes of the Hamiltonian constraint. Partial results are given in the Appendix; for now, I will avoid this issue, and merely use $|\Psi|^{2}$ to determine relative probabilities, with the understanding that the inner product measure may give corrections.

It is worth reiterating that $\Psi$ is a functional of the metric, not a function of space. Its role is to determine probabilities of configurations $(h, f)$. Of course, the probability of any given metric occurring will depend on its spatial form, and we shall see that a wave function can imply "a high probability for metrics with spatial characteristic X." But this will only make sense if the "spatial characteristic X" is expressed in a diffeomorphism-invariant way, one that does not refer to any particular values of the coordinates. 


\subsection{The probability current}

As a Schrödinger-type equation, (5.9) should also admit a probability current. Recall that in the WKB approximation in ordinary quantum mechanics, the probability current distinguishes genuinely time-independent configurations (e.g., bound states) from steady state descriptions of a secretly time-dependent configurations (e.g., plane waves scattering off a potential barrier). We shall see that the same is true here.

It is easy to see that the current

$$
J[f, h ; x]=\frac{3 i \kappa^{2}}{64 \pi^{2}}\left(\Psi^{*} \frac{1}{f^{2}} \frac{\delta \Psi}{\delta h}-\Psi \frac{1}{f^{2}} \frac{\delta \Psi^{*}}{\delta h}\right) .
$$

obeys a sort of continuity equation on midisuperspace,

$$
\frac{d\left(\Psi^{*} \Psi\right)}{d T}+\frac{1}{f^{2}} \frac{\delta J}{\delta h}=0
$$

But $J$ is not an observable: it is not annihilated by the momentum constraint $\hat{\mathscr{P}}$. We can project $J$ onto reduced midisuperspace by taking a volume average, or equivalently forming a group average over the spatial diffeomorphisms [26],

$$
\langle J\rangle=\frac{1}{V} \int d^{3} x h f^{2} J,
$$

but this average no longer obeys an obvious continuity equation.

To do better, consider the functions

$$
\varphi_{n}(\psi)=\exp \left\{\frac{8 \pi^{2} i n}{V} \int^{\psi} f^{2} h d \psi^{\prime}\right\}
$$

These are orthogonal and complete:

$$
\begin{aligned}
& \int h f^{2} \varphi_{m}^{*} \varphi_{n} d \psi=\frac{V}{4 \pi} \delta_{m n}, \\
& \sum_{n} \varphi_{n}^{*}\left(\psi^{\prime}\right) \varphi_{n}(\psi)=\frac{V}{4 \pi h f^{2}} \delta\left(\psi-\psi^{\prime}\right) .
\end{aligned}
$$

If we now project

$$
D_{n}=\frac{1}{V} \int d^{3} x h f^{2} \varphi_{n}^{*}\left(\frac{1}{f^{2}} \frac{\delta}{\delta h}\right), \quad J_{n}=\int d^{3} x h f^{2} \varphi_{n} J
$$

a bit of work shows that

$$
\sum_{n} D_{n} J_{n}=\frac{1}{V} \int d^{3} x h f^{2}\left(\frac{1}{f^{2}} \frac{\delta J}{\delta h}\right)=-\frac{d\left(\Psi^{*} \Psi\right)}{d T},
$$

where the last equality comes from (5.12). $D_{n}$ and $J_{n}$ are spatial invariants, that is, that they are annihilated by the momentum constraint The $D_{n}$ may be viewed as the components of the gradient in our (infinite-dimensional) midisuperspace, making (5.17) a standard continuity equation.

\footnotetext{
${ }^{\ddagger} D_{n}$ and $J_{n}$ are not annihilated by the Hamiltonian constraint; a further projection may be needed to construct spacetime invariants, but that lies beyond the scope of this paper.
} 


\section{Stationary states and the WKB approximation}

We next look for stationary states

$$
\Psi[f, h ; T]=\tilde{\Psi}[f, h] e^{-i E T} .
$$

For states of this form, the Wheeler-DeWitt equation (5.9) becomes

$$
\left[\frac{\kappa^{2}}{64 \pi^{2}}\left(\frac{1}{f^{2}} \frac{\delta}{\delta h}\right)^{2}+\frac{1}{\kappa^{2}} \frac{1}{f^{2}}\left(\frac{f^{\prime 2}}{h^{2}}-1\right)+\frac{\tilde{\Lambda}}{3 \kappa^{2}}+\frac{\gamma}{\kappa^{2} f^{3}}\right] \tilde{\Psi}=0,
$$

where

$$
\tilde{\Lambda}=\Lambda-\kappa^{2} E
$$

This equation is identical to the original gravitational Hamiltonian constraint, except that the cosmological constant is shifted by the energy, again reminiscent of unimodular gravity [23]. This shift in $\Lambda$ is physical - it is a backreaction of our "cloud of clocks" on the spacetime - and to see purely gravitational properties, we should limit ourselves to states with relatively small energies. For a Planck-scale $\Lambda$, though, this is a rather mild restriction.

To better understand these states, let us consider a WKB approximation,

$$
\tilde{\Psi}=A e^{i S} .
$$

The first order WKB equation,

$$
\frac{\kappa^{2}}{64 \pi^{2}}\left(\frac{\delta S}{\delta h}\right)^{2}=\frac{1}{\kappa^{2}} f^{2}\left(\frac{f^{\prime 2}}{h^{2}}-1\right)+\frac{\tilde{\Lambda}}{3 \kappa^{2}} f^{4}+\frac{\gamma}{\kappa^{2}} f
$$

can be solved exactly:

$$
S=\frac{8 \pi}{\kappa^{2}} \int d \psi \sigma[h, f ; \psi] f f^{\prime}\left\{\sqrt{1+\beta h^{2}}-\tanh ^{-1} \sqrt{1+\beta h^{2}}\right\},
$$

where

$$
\beta=\frac{f^{2}}{f^{\prime 2}}\left(\frac{\tilde{\Lambda}}{3}-\frac{1}{f^{2}}+\frac{\gamma}{f^{3}}\right)
$$

and $\sigma$ is a functional of $h$ and $f$ and a function of $\psi$ such that

$$
\begin{cases}\sigma^{2}=1 & \text { almost everywhere } \\ \partial_{\psi} \sigma=0 & \text { unless } 1+\beta h^{2}=0 .\end{cases}
$$

The factor $\sigma$ requires a bit of explanation. As always in the WKB approximation, the phase $S$ is determined only up to sign. But eqn. (6.5) holds pointwise, and thus admits solutions in which this sign, $\sigma$, can vary with $f, h$, and $\psi$. The choice is not completely free, though: we must 
still require that $S$ be annihilated by the momentum constraint. If the position dependence of $\sigma$ only arose implicitly from its dependence on $h$ and $f$, then $S$ would be of the form (5.5), and we would automatically have $\hat{\mathscr{P}} S=0$. But any explicit dependence of $\sigma$ on $\psi$ introduces a new term in $\hat{\mathscr{P}} S$, proportional to $\left(1+\beta h^{2}\right)^{1 / 2} \partial_{\psi} \sigma$. The constraints require this term to vanish, leading to the second condition in (6.8).

To understand the physical significance of this condition, we can rewrite (6.7) as

$$
1+\beta h^{2}=\frac{\kappa^{4} \tilde{P}^{2}}{f^{2} f^{\prime 2}}
$$

where

$$
\tilde{P}=\frac{1}{\kappa^{2}}\left[f^{2} h^{2}\left(\frac{f^{\prime 2}}{h^{2}}-1\right)+\frac{\tilde{\Lambda}}{3} f^{4} h^{2}+\gamma f h^{2}\right]^{1 / 2} .
$$

Comparing to (2.8), we see that $\tilde{P}$ is essentially the classical momentum $\pi^{\psi}{ }_{\psi}$, though with a shifted cosmological constant. Hence the sign of $S$ can change only when the $\tilde{P}$ goes through zero, the quantum version of the the classical sewing condition of section 3 , There is also an analog of the inequality (2.10) for $\gamma$. For large $f, \beta$ is positive, while for $\tilde{P}$ to go through zero, $\beta$ must be negative. But (6.7) involves the same cubic as (3.3), and $\beta$ can change sign only if the inequality (2.10) is satisfied, albeit again with a shifted $\Lambda$.

This means, in particular, that for suitable $\gamma$, the quantum theory allows such sewing. More precisely, let $(h, f)$ be a metric configuration that, as a classical metric, describes layers joined by necks in which the extrinsic curvature goes through zero. A choice of $\sigma$ for this configuration is then equivalent to a choice of the sign of the extrinsic curvature in each layer. Just as this sign is not determined classically by the constraints, it is not determined quantum mechanically by the Wheeler-DeWitt equation: different choices of $\sigma$ give different wave functions $\Psi_{\sigma}$.

We can now explore our WKB wave functions in various regions of midisuperspace. First, consider the case of large $f$-that is, specialize to metrics in which $f$ is large in some region of space, and look at the contribution of that spatial region to the integral (6.6). In such a region, $\tilde{P} \gg 0$, so the sign $\sigma$ is fixed, and $\beta$ is dominated by the cosmological constant, yielding

$$
S \sim \frac{2}{\kappa^{2}} \sigma\left(\frac{\tilde{\Lambda}}{3}\right)^{1 / 2} V
$$

The mean curvature operator (5.3b) - the local Hubble constant - then has a simple action,

$$
\hat{K} e^{i S} \approx 3 \sigma\left(\frac{\tilde{\Lambda}}{3}\right)^{1 / 2} e^{i S}
$$

This is ordinary de Sitter behavior, and, as expected, the sign $\sigma$ determines whether a spatial region is expanding or contracting. The probability current (5.16) provides similar information:

$$
J_{n}=-\frac{3 V}{4 \pi} \sigma\left(\frac{\tilde{\Lambda}}{3}\right)^{1 / 2} \delta_{n 0},
$$


so $\sigma$ determines the direction of flow of probability. This is not unlike the WKB approximation in ordinary quantum mechanics, where a plane wave $e^{i k x}$ is formally a stationary state, but the probability current reveals a hidden dynamics.

But the wave function does not have its support only on such de Sitter-like regions. Consider the contribution of a multilayered "foamy" region. From (5.3b) and (5.4), the mean curvature acts on diffeomorphism-invariant states as

$$
\hat{K}=\frac{i \kappa^{2}}{8 \pi} \frac{1}{f^{2} f^{\prime}} \partial_{\psi}\left(f \frac{\delta}{\delta h}\right)
$$

yielding

$$
\hat{K} e^{i S}=\frac{1}{f^{2} f^{\prime}} \partial_{\psi}\left(\frac{f^{2} f^{\prime}}{h} \sigma \sqrt{1+\beta h^{2}}\right) e^{i S}=\frac{\kappa^{2}}{f^{2} f^{\prime}} \partial_{\psi}\left(\frac{f}{h} \sigma \tilde{P}\right) e^{i S}
$$

As expected, the WKB wave function is not an eigenfunction of $\hat{K}$. But in for foamy regions of midisuperspace, it is almost an eigenfunction of the spatially averaged mean curvature. Indeed,

$$
\left(\frac{1}{V} \int d^{3} x \sqrt{q} \hat{K}\right) e^{i S}=-\frac{4 \pi \kappa^{2}}{V} \int d \psi \frac{f}{h}\left(\frac{h}{f^{\prime}}\right)^{\prime} \sigma \tilde{P} e^{i S}
$$

For a typical wave function, evaluated at a typical multilayered metric, the contribution of each layer will come with a random sign $\sigma$. Hence for a configuration with many layers, we expect extensive cancellation - just as in the classical case, the averaged mean curvature will be much smaller than it would be for a single de Sitter region.

The evaluation of the probability current leads to an identical conclusion, perhaps even more clearly. The current is

$$
J_{n}=-\frac{3 \kappa^{2}}{4} \int d^{3} x \sigma \tilde{P} \varphi_{n}
$$

and for foamy metrics the right-hand side will again average to a very small number. Our wave functions are thus stationary not only in the sense that they are independent of $T$, but also in the sense that there is very little flow of probability within midisuperspace, so initial foamy structures will tend to be preserved.

Of course, this leaves open the question of how common such multilayered foamy configurations are. An answer requires the next order WKB approximation, which we will turn to shortly. Meanwhile, there are a few other features of (6.6) that deserve future exploration:

1. The integrand in (6.6) diverges when $\beta h^{2}=0$. Comparing (6.7) to (2.9), we might suspect this to occur at some sort of horizon. This is correct: the vanishing of $\beta h^{2}$ marks the location of a marginally trapped sphere, a midisuperspace version of the trapped surfaces that appear in the more general connected sum analysis [14].

2. The integrand vanishes at $1+\beta h^{2}=0$, the classical neck. But it also has zeroes at $1+\beta h^{2}=-z_{n}^{2}$, where $z_{n}=\tan z_{n}$. These are in a classically forbidden region, but in principle they are additional sites at which expanding and contracting regions can join. 
3. When $1+\beta h^{2}>0, \tanh ^{-1} \sqrt{1+\beta h^{2}}$ has a constant imaginary part. In a region between two successive zeroes of $1+\beta h^{2}$, though, $\sigma$ is constant, and the imaginary part of $S$ is

$$
\frac{4 \pi^{2} i}{\kappa^{2}} \int_{\psi_{1}}^{\psi_{2}} d \psi \sigma f f^{\prime}=\frac{2 \pi^{2} i}{\kappa^{2}} \sigma\left[f^{2}\left(\psi_{2}\right)-f^{2}\left(\psi_{1}\right)\right]
$$

with $1+\beta h^{2}=0$ at $\psi_{1}$ and $\psi_{2}$. We saw earlier that at a "classical" zero of $1+\beta h^{2}$, the value of $f$ is fixed by (3.3), so the contributions of the two endpoints cancel. It is less clear what happens at the nonclassical zeroes $1+\beta h^{2}=-z_{n}^{2}$.

\section{Next order WKB}

To say more about probabilities for "foamy" spacetimes, we will need to go to the next order in the WKB approximation (6.4),

$$
2 \frac{\delta S}{\delta h} \frac{\delta A}{\delta h}+A \frac{\delta^{2} S}{\delta h^{2}}=0 .
$$

This equation involves two functional derivatives at a single point, leading to a well known divergence. For our midisuperspace model, we can write

$$
S=\int d \psi L
$$

where $L$ depends on $h$ but not $h^{\prime}$. Then

$$
\frac{\delta S}{\delta h(\psi)}=\frac{\partial L}{\partial h}(\psi), \quad \frac{\delta^{2} S}{\delta h(\psi) \delta h\left(\psi^{\prime}\right)}=\frac{\partial^{2} L}{\partial h^{2}}(\psi) \delta\left(\psi-\psi^{\prime}\right),
$$

giving a factor $\delta(0)$ at $\psi=\psi^{\prime}$.

There are several proposals for regulating this infinite factor. In the original formulation of the Wheeler-DeWitt equation [25, DeWitt suggested that such $\delta(0)$ factors should be set to zero. Here, that choice would make the first order WKB approximation exact. A "volume average regularization" [27], applied to this simple case, replaces $\delta\left(\psi-\psi^{\prime}\right)$ by

$$
\frac{h}{\ell} \int d \psi^{\prime} \delta\left(\psi-\psi^{\prime}\right)
$$

where $\ell=\int d \psi h$ is the length of the spatial $S^{1}$ factor. Heat kernel regularization [28] replaces the factor $\delta\left(\psi-\psi^{\prime}\right)$ by a heat kernel $K\left(\psi, \psi^{\prime} ; s\right)$. In the $s \rightarrow 0$ limit, $K\left(\psi, \psi^{\prime} ; s\right)$ becomes a delta function, but if one first takes $\psi \rightarrow \psi^{\prime}$, the divergences appear as a set of terms involving inverse powers of $s^{1 / 2}$, which can be individually regularized. In the present context, the outcome is almost the same as the volume average: $\delta\left(\psi-\psi^{\prime}\right)$ becomes

$$
\alpha h \int d \psi^{\prime} \delta\left(\psi-\psi^{\prime}\right)
$$

where $\alpha$ is an undetermined constant with dimensions of inverse length. 


\subsection{Heat kernel regularization}

Let us first consider heat kernel regularization. The second order WKB equation becomes

$$
\frac{1}{A} \frac{\delta A}{\delta h}=-\frac{\alpha h}{2}\left(\frac{\partial L}{\partial h}\right)^{-1} \frac{\partial^{2} L}{\partial h^{2}}
$$

or with $S$ as in (6.6),

$$
\frac{1}{A} \frac{\delta A}{\delta h}=\frac{\alpha}{2} \frac{1}{1+\beta h^{2}}
$$

This can be solved in closed form:

$$
A=\exp \left\{\frac{\alpha}{2} \int d \psi \frac{1}{\sqrt{\beta}} \tan ^{-1}(\sqrt{\beta} h)\right\} .
$$

We can now look at relative probabilities for behaviors of the metric - that is, at features of the functions $(h, f)$ that lead to comparatively small or large vales of the amplitude $A$.

- When $\beta h^{2}$ is large, the integrand in (7.6) is small, since $\tan ^{-1}(\sqrt{\beta} h)$ is bounded by $\pi / 2$. By (6.9), this implies that metrics with large regions of high extrinsic curvature are suppressed. If we abbreviate

$$
\left|\frac{\kappa^{2} \tilde{P}}{f f^{\prime}}\right|=\sqrt{1+\beta h^{2}}=\Delta
$$

then for a region of length $\ell_{1}$,

$$
\left.\ln A\right|_{\Delta \gg 1} \sim \frac{\alpha \pi}{4}\left\langle\Delta^{-1}\right\rangle \ell_{1} \ll \frac{\alpha \ell_{1}}{2},
$$

where the angle brackets denote a spatial average.

- When $\beta h^{2}$ is small but positive, it is still the case that $\tan ^{-1}(\sqrt{\beta} h)<\sqrt{\beta} h$, so

$$
\left.\ln A\right|_{\Delta \sim 1} \lesssim \frac{\alpha \ell_{1}}{2}
$$

- When $\beta h^{2}<0$,

$$
\frac{\alpha}{2} \int d \psi \frac{1}{\sqrt{\beta}} \tan ^{-1}(\sqrt{\beta} h)=\frac{\alpha}{4} \int d \psi \frac{1}{\sqrt{|\beta|}} \ln \left(\frac{1+\sqrt{|\beta|} h}{1-\sqrt{|\beta|} h}\right),
$$

and the integral receives large contributions when $1+\beta h^{2} \sim 0$, that is, from the "necks" of the preceding section. Again by (6.9), in regions of small $\tilde{P}$,

$$
\frac{\alpha}{2} \int d \psi \frac{1}{\sqrt{\beta}} \tan ^{-1}(\sqrt{\beta} h) \approx \frac{\alpha}{2} \int d \psi h \ln \left(\frac{2 f f^{\prime}}{\kappa^{2}|\tilde{P}|}\right) .
$$


Classically, we saw in section 3 that $\tilde{P} \sim \psi^{3}$ near a neck, while $f^{\prime} \sim \psi$, so the integrand goes as $h \ln (a / h \psi)$ for some parameter $a$. The integral is maximum when integrated over a region of length $\ell_{1} \sim a$, and yields

$$
\left.\ln A\right|_{\Delta \ll 1} \lesssim \frac{\alpha \ell_{1}}{2}
$$

(The dependence on the parameter $a$ imposes no further limits. By section 3 ,

$$
\left.a \sim\left(f^{2} D^{3} K\right)^{-1 / 2}\right|_{\psi=0}
$$

where $K$ is a component of the extrinsic curvature, $D$ is the invariant derivative (5.5), and $f(0)$ is fixed by (3.3). If the next order of the expansion (3.4) involves only constants of order one, then $a \sim f_{0}$, the characteristic size of the neck. But $f_{0}$ itself depends on the integration constant $\gamma$, and can range from 0 to $\sqrt{3 / \Lambda}$. Moreover, there is no strong reason to demand that the expansion (3.4) have coefficients of order one; if extrinsic curvatures remain small near a neck, $a$ can be large.)

Combining these results, we see that the wave function strongly disfavors spaces with large regions of high extrinsic curvature (large $\Delta$ ), while giving roughly equal weight to metrics with regions of relatively small intrinsic curvature near the start of a "neck" (trapped surfaces $\Delta=1$ ) and regions of very small extrinsic curvature where the sign of the expansion can change $(\Delta=0)$. Note that at this order, there is no limit to the proper length $\ell$. Since the integrand in (7.6) is positive, this suggests an infrared divergence. As observed in section 5, though, we have not yet accounted for the measure in the inner product, so it is premature to draw too firm a conclusion.

We can also ask how this higher order WKB term affects the probability current. For $\beta h^{2}>-1, A$ is real, so it merely multiplies the lowest order current (5.11) by $A^{2}$. For $\beta h^{2}<-1$, the exponent (7.10) becomes complex, but the imaginary part is independent of $h$, and does not contribute to the current. The moments (6.17) thus become

$$
J_{n}=-\frac{3 \kappa^{2}}{4} \int d^{3} x \sigma|A|^{2} \tilde{P} \varphi_{n} .
$$

\subsection{Volume regularization}

We can now repeat the argument using volume regularization. Eqn. (7.5) becomes

$$
\frac{1}{A} \frac{\delta A}{\delta h}=\frac{1}{2 \ell} \frac{1}{1+\beta h^{2}},
$$

and it is tempting to guess a solution

$$
A=\exp \left\{\frac{1}{2 \ell} \int d \psi \frac{1}{\sqrt{\beta}} \tan ^{-1}(\sqrt{\beta} h)\right\} .
$$


This doesn't quite work, though: because of the metric dependence of $\ell$,

$$
\frac{1}{A} \frac{\delta A}{\delta h}=\frac{1}{2 \ell} \frac{1}{1+\beta h^{2}}-\frac{1}{2 \ell^{2}} \int d \psi \frac{1}{\sqrt{\beta}} \tan ^{-1}(\sqrt{\beta} h) .
$$

In fact, (7.14) is not integrable: for any putative solution,

$$
\frac{\delta^{2} A}{\delta h(\psi) \delta h\left(\psi^{\prime}\right)} \neq \frac{\delta^{2} A}{\delta h\left(\psi^{\prime}\right) \delta h(\psi)} .
$$

The amplitude (7.15) is, however, a good approximate solution, since it follows from the preceding section that the extra term in (7.16) is of order $1 / \ell$. It might be possible to view this extra piece as a counterterm in the WKB equation (7.1) - it amounts to adding a term proportional to $\frac{\delta S}{\delta h}$ to the divergent $\frac{\delta^{2} S}{\delta h^{2}}$ - but it would be a rather peculiar one.

If we ignore this issue, the conclusions from volume regularization are almost identical to those from heat kernel regularization. The only difference is that the prefactor $\alpha$ now becomes $1 / \ell$, providing a natural infrared cutoff.

\section{Conclusions and next steps}

The space of locally spherically symmetric three-geometries is surprisingly rich. It includes configurations that exhibit spacetime foam: spaces with multiple layers with different geometries, joined by connected sums. The constraints determine the extrinsic curvature only up to a sign, and a typical multilayered configuration includes both expanding and contracting regions. While the these spacetimes are certainly not realistic models of our Universe, they are qualitatively very similar to the more general foamy geometries discussed in [2].

This structure persists in the quantum theory. By using the "dust time" of Brown and Kuchar, in which physical time is proper time along a congruence of timelike geodesics, we can reduce the Wheeler-DeWitt equation to a Schrödinger-type equation, and search for stationary states. These states have a sign ambiguity in their phase that closely mimics the classical ambiguity in the sign of the extrinsic curvature. When evaluated on a multilayer metric, this sign $\sigma$ can change at precisely the locations that the sign of the extrinsic curvature can change classically.

As we know from quantum mechanics, some care must be taken in interpreting such states. A stationary state may imply time independence, but it may instead signify a steady state flow of probability, as in a scattering state. Indeed, here one can construct a state in which the sign $\sigma$ in (6.6) is the same for every layer. The probability current is then large, proportional to the de Sitter expansion $\sqrt{\Lambda / 3}$. But for a typical state, the current (7.13), evaluated at a typical multilayered geometry, will be very small: the foamy structure will tend to reproduce itself in a nearly steady state. This lends support to the proposal of [2] that a cosmological constant might be "hidden" in spacetime foam.

How common are multilayered foamy configurations? The second order WKB approximation has regularization ambiguities, but standard choices such as heat kernel regularization indicate that they are at least fairly probable. Indeed, geometries with large regions of high extrinsic curvature are strongly disfavored, while necks that occur in connected sums of layers 
are favored. Note, though, that the quantum version of the inequality (2.10) depends, via (6.3), on the state, so for fixed $\gamma$ the prevalence of foamy configurations is state dependent.

There are, of course, further questions that must be answered before we can be confident in these conclusions. Perhaps the most important is the problem of the measure for the inner product on midisuperspace. I have been using the "naive Schrödinger interpretation" for $|\Psi|^{2}$ [15], which implicitly assumes that the correct inner product is just a functional integral over $h$ and $f$. But this functional integral must be gauge fixed to account for the invariances generated by the constraints, and the resulting Faddeev-Popov determinants may affect probabilities [24]. Partial results in this direction are described in the Appendix. Crucially, the Faddeev-Popov determinant is independent of the sign of the extrinsic curvature, and will not change the cancellation between expanding and contracting regions.

It would also be useful to move beyond stationary states and look at the behavior of wave packets. Our choice of time makes this a bit difficult: our "cloud of clocks" back-reacts on the geometry, and for this to be unimportant we must restrict the energy to be small (on the scale set by $\Lambda$ ). This means wave packets cannot be too sharply peaked. Ideally, one would avoid this by choosing a purely gravitational "internal time," but such a parameter seems very hard to find in a spacetime containing both expanding and contracting regions. A more careful study of the role of the integration constant $\gamma$ would also be valuable. In particular, it is not currently clear whether different choices of $\gamma$ form superselection sectors.

A number of straightforward extensions of this work should be possible. One could apply the same techniques to a spatial topology $S^{3}$, by again starting with a manifold $[0,1] \times S^{2}$ but now shrinking each boundary to a point. Beyond that, though, Morrow-Jones and Witt have shown that local spherical symmetry allows a huge variety of topologies [5], although a detailed analysis would require much more complicated boundary conditions. An extension to $\Lambda<0$ should also be easy; most of the computations will be unchanged, though the qualitative results are likely to be quite different.

It would also be interesting to look at the "reduced phase space" quantization of this model, in which the reparametrization invariance of $\psi$ is gauge fixed and the momentum constraint is eliminated classically. Whether such a quantization is equivalent to the Dirac quantization method used here is an open question, involving subtleties in operator ordering and the choice of inner product [29, 30], but it is certainly worth exploring. Unfortunately, it is also a bit harder than it might appear. Following [5, we can nearly fix the reparametrization invariance by redefining $\psi$ to make $h$ constant on the initial slice. But the integral $\int h d \psi$ is invariant, and leaves us with an extra variable (which is also classically time dependent). The resulting Hamiltonian constraint then contains a mixture of ordinary and functional derivatives, and becomes technically quite complicated.

Finally, there may be another somewhat orthogonal approach to these questions. One way to view spacetime foam in a universe with large $\Lambda$ is to consider the nucleation of contracting bubbles in expanding regions, and expanding bubbles in contracting regions. It is then natural to ask whether there are instantons that mediate such processes. In the context of our locally spherically symmetric midisuperspace, the question is whether there are Euclidean solutions joining a space with $n$ layers to one with $n+1$ layers. Some very preliminary calculations show no obstruction to such solutions, but much more work is needed. 


\section{Acknowledgments}

This work was supported in part by Department of Energy grant DE-FG02-91ER40674. I would also like to thank the Quantum Gravity Unit of the Okinawa Institute of Science and Technology (OIST), where part of the work was completed, for their hospitality.

\section{Appendix. A note on the inner product}

As noted in the conclusion, a crucial remaining problem is to find the correct inner product on midisuperspace. Our wave functions are functions of $f$ and $h$, and the naive inner product is simply a functional integral $\int[d f][d h]$. This is not quite right, though, for two reasons.

First, our symmetry-reduced midisuperspace still has a residual diffeomorphism invariance, reparametrizations of $(t, \psi)$. The $t$ reparametrizations are fixed by the dust time gauge $t=T$ of section 4, but the $\psi$ reparametrizations remain, and the integral will count identical configurations an infinite number of times. Second, these gauge fixing of the residual diffeomorphisms will induce a Faddeev-Popov determinant, which must also be taken into account.

Locally, the $\psi$ reparametrizations can be fixed by setting $\partial_{\psi} h=0$, as was done in [5]. Note that the total length $\int d \psi h$ is diffeomorphism invariant, so the constant value of $h$ is fixed. Whether this choice can be made globally over the whole midisuperspace - that is, whether there are Gribov problems [31,32] - is a more difficult question.

The Faddeev-Popov determinants are best understood as Jacobians in the functional integral, as described in [33, 34]. (For an early version, see [35.) Let $\phi$ be a generic field, and define an inner product $\langle\delta \phi, \delta \phi\rangle$ on the tangent space to the space of fields. The standard normalization for the path integral is

$$
\int[d \phi] e^{-\langle\delta \phi, \delta \phi\rangle}=1 .
$$

But now suppose $\phi$ has a gauge symmetry $\phi \rightarrow^{\eta} \phi$, labeled by some parameter $\eta$. We can change variables in the functional integral to $(\bar{\phi}, \eta)$, where $\bar{\phi}$ is a gauge-fixed field. Geometrically, $\eta$ parametrizes gauge orbits, while $\bar{\phi}$ parametrizes a cross section that intersects each orbit once. (Whether such a cross section exists or not is the Gribov problem.) Then

$$
\int[d \phi] e^{-\langle\delta \phi, \delta \phi\rangle}=\int[d \bar{\phi}][d \eta] J e^{-\langle\delta \bar{\phi}, \delta \bar{\phi}\rangle} e^{-\left\langle\delta_{\eta} \phi, \delta_{\eta} \phi\right\rangle}=\int[d \eta] J e^{-\left\langle\delta_{\eta} \phi, \delta_{\eta} \phi\right\rangle}=1,
$$

where $J$ is a Jacobian coming from the change of variables. This Jacobian is the Faddeev-Popov determinant.

For us, the fields are the metric $q_{i j}$ and the "dust" field $T$, with natural inner products

$$
\begin{aligned}
\langle\delta g, \delta g\rangle & =\int d^{3} x \sqrt{q} q^{i j} q^{k \ell} \delta q_{i k} \delta q_{j \ell}=4 \pi \int d \psi h f^{2}\left[4\left(\frac{\delta h}{h}\right)^{2}+8\left(\frac{\delta f}{f}\right)^{2}\right] \\
\langle\delta T, \delta T\rangle & =\int d x^{3} \sqrt{q}(\delta T)^{2}=4 \pi \int d \psi h f^{2}(\delta T)^{2}
\end{aligned}
$$


The transformations generated by the constraints (2.1a $-(2.1 \mathrm{~b})$ involve two parameters $\left(\xi, \xi^{\perp}\right)$, a $\psi$ reparametrization and a "surface deformation" (basically a $t$ reparametrization). It is straightforward to check that

$$
\frac{\delta_{\eta} h}{h}=\xi^{\prime}+\frac{h^{\prime}}{h} \xi+K_{\psi}^{\psi} \xi^{\perp}, \quad \frac{\delta_{\eta} f}{f}=\frac{f^{\prime}}{f} \xi+K^{\theta}{ }_{\theta} \xi^{\perp}, \quad \delta_{\eta} T=\xi^{\perp},
$$

where $K^{\psi}{ }_{\psi}$ and $K^{\theta}{ }_{\theta}$ are the extrinsic curvatures (2.5). Thus the inner product $\left\langle\delta_{\eta} \phi, \delta_{\eta} \phi\right\rangle$ becomes

$$
\left\langle\delta_{\eta} \phi, \delta_{\eta} \phi\right\rangle=4 \pi \int d \psi h f^{2}\left[4\left(\xi^{\prime}+\frac{h^{\prime}}{h} \xi+K_{\psi}^{\psi} \xi^{\perp}\right)^{2}+8\left(\frac{f^{\prime}}{f} \xi+K^{\theta}{ }_{\theta} \xi^{\perp}\right)^{2}+\left(\xi^{\perp}\right)^{2}\right] .
$$

Let us define

$$
\tilde{K}^{2}=K^{i}{ }_{j} K^{j}{ }_{i}=K^{\psi}{ }^{2}+2 K^{\theta}{ }^{2}
$$

Then the integrand in (A.5) has the form

$$
\left(1+4 \tilde{K}^{2}\right)\left(\xi^{\perp}\right)^{2}+\left[8\left(\xi^{\prime}+\frac{h^{\prime}}{h} \xi\right) K_{\psi}^{\psi}+16 \frac{f^{\prime}}{f} \xi K_{\theta}^{\theta}\right] \xi^{\perp}+\ldots
$$

where the remaining terms are independent of $\xi^{\perp}$. We can now complete the square, setting

$$
\bar{\xi}^{\perp}=\xi^{\perp}+4\left(1+4 \tilde{K}^{2}\right)^{-1}\left[\left(\xi^{\prime}+\frac{h^{\prime}}{h} \xi\right) K_{\psi}^{\psi}+2 \frac{f^{\prime}}{f} \xi K_{\theta}^{\theta}\right] .
$$

After some integrations by parts, we obtain an expression of the form

$$
\left\langle\delta_{\eta} \phi, \delta_{\eta} \phi\right\rangle=4 \pi \int d \psi h f^{2}\left[\left(\bar{\xi}^{\perp}\right)^{2}+B \xi\left(D^{2}+V\right) \xi\right]
$$

where $D$ is the derivative (5.5). The coefficients $B$ and $V$ are complicated functions of $f$ and $h$, but they are independent of $\bar{\xi}^{\perp}$. Crucially, they are also quadratic in the extrinsic curvature, and hence invariant under $K^{i}{ }_{j} \rightarrow-K^{i}{ }_{j}$. The Jacobian (A.2) thus takes the form

$$
J=\operatorname{det}^{1 / 2}\left|B\left(D^{2}+V\right)\right|,
$$

and is independent of the sign of the extrinsic curvature. Hence the inner product does not break the time-reversal symmetry that produces cancellations between regions with different signs $\sigma$.

It may be possible to obtain further information about $J$ through zeta function methods. In particular, the scaling behavior could be important in understanding the possible infrared divergences discussed in section 7.1. For now, though, this lies beyond the scope of this paper. 


\section{References}

[1] J. A. Wheeler, "Geons," Phys. Rev. 97 (1955) 511.

[2] S. Carlip, "Hiding the Cosmological Constant," Phys. Rev. Lett. 123 (2019) 131302, arXiv:1809.08277.

[3] P. T. Chrusciel, J. Isenberg, and D. Pollack, "Gluing initial data sets for general relativity," Phys. Rev. Lett. 93 (2004) 081101, arXiv:gr-qc/0409047.

[4] P. T. Chrusciel, J. Isenberg, and D. Pollack, "Initial data engineering," Commun. Math. Phys. 257 (2005) 29, arXiv:gr-qc/0403066.

[5] J. Morrow-Jones and D. M. Witt, "Inflationary initial data for generic spatial topology," Phys. Rev. D 48 (1993) 2516.

[6] K. Schleich and D. M. Witt, "Designer de Sitter Spacetimes," Can. J. Phys. 86 (2008) 591. arXiv:0807.4559.

[7] K. Schleich and D. M. Witt, "What does Birkhoff's theorem really tell us?" arXiv:0910.5194.

[8] J. D. Brown and K. V. Kuchař, "Dust as a standard of space and time in canonical quantum gravity," Phys. Rev. D51 (1995) 5600, arXiv:gr-qc/9409001.

[9] J. D. Brown and D. Marolf, "On relativistic material reference systems," Phys. Rev. D53 (1996) 1835, arXiv:gr-qc/9509026.

[10] V. Husain and T. Pawlowski, "Time and a physical Hamiltonian for quantum gravity," Phys. Rev. Lett. 108 (2012) 141301, arXiv:1108.1145.

[11] S. Carlip, General Relativity (Oxford University Press, 2019).

[12] K. Lake and R. C. Roeder, "Effects of a Nonvanishing Cosmological Constant on the Spherically Symmetric Vacuum Manifold," Phys. Rev. D 15 (1977) 3513.

[13] K. Schleich and D. M. Witt, "A simple proof of Birkhoff's theorem for cosmological constant," J. Math. Phys. 51 (2010) 112502, arXiv:0908.4110.

[14] M. Burkhart and D. Pollack, "Causal geodesic incompleteness of spacetimes arising from IMP gluing," arXiv:1907.00295.

[15] K. V. Kuchař, "Time and Interpretations of Quantum Gravity," Int. J. Mod. Phys. D20 (2011) 3.

[16] C. J. Isham, "Canonical quantum gravity and the problem of time," NATO Sci. Ser. C 409 (1993) 157, arXiv:gr-qc/9210011.

[17] C. W. Misner, "Quantum cosmology 1," Phys. Rev. 186 (1969) 1319.

[18] N. Ó Murchadha, C. Soo, and H.-L. Yu, "Intrinsic time gravity and the Lichnerowicz-York equation," Class. Quant. Grav. 30 (2013) 095016, arXiv:1208.2525.

[19] Y. Choquet-Bruhat and J. York, "The Cauchy problem," in General Relativity and Gravitation I, edited by A. Held (New York: Plenum, 1980).

[20] M. Kleban and L. Senatore, "Inhomogeneous Anisotropic Cosmology," JCAP 10 (2016) 022, arXiv:1602.03520.

[21] L. Andersson, G. J. Galloway, and R. Howard, "The Cosmological time function," Class. Quant. Grav. 15 (1998) 309, arXiv:gr-qc/9709084. 
[22] K. V. Kuchar and C. G. Torre, "Gaussian reference fluid and interpretation of quantum geometrodynamics," Phys. Rev. D 43 (1991) 419.

[23] W. G. Unruh, "A Unimodular Theory of Canonical Quantum Gravity," Phys. Rev. D 40 (1989) 1048.

[24] R. P. Woodard, "Enforcing the Wheeler-de Witt Constraint the Easy Way," Class. Quant. Grav. $10(1993) 483$.

[25] B. S. DeWitt, "Quantum Theory of Gravity 1. The Canonical Theory," Phys. Rev. 160 (1967) 1113.

[26] D. Marolf, "Group averaging and refined algebraic quantization: Where are we now?" in Proc. of the 9th Marcel Grossmann Meeting, edited by V. G. Gurzadian, R. T. Jantzen, and R. Ruffini (Singapore: Wold Scientific, 2002), arXiv:gr-qc/0011112.

[27] J. C. Feng, "Volume average regularization for the Wheeler-DeWitt equation," Phys. Rev. D 98 (2018) 026024, arXiv:1802.08576.

[28] T. Horiguchi(, K. Maeda(, and M. Sakamoto, "Analysis of the Wheeler-DeWitt equation beyond Planck scale and dimensional reduction," Phys. Lett. B 344 (1995) 105, arXiv:hep-th/9409152.

[29] G. Kunstatter, "Dirac versus reduced quantization: A Geometrical approach," Class. Quant. Grav. 9 (1992) 1469.

[30] R. J. Epp, "Curved space quantization: Toward a resolution of the Dirac versus reduced quantization question," Phys. Rev. D 50 (1994) 6578.

[31] V. N. Gribov, "Quantization of Nonabelian Gauge Theories," Nucl. Phys. B 139 (1978) 1.

[32] M. Schon and p. Hajicek, "Topology of Quadratic Superhamiltonians," Class. Quant. Grav. 7 (1990) 861.

[33] J. Polchinski, "Evaluation of the One Loop String Path Integral," Commun. Math. Phys. 104 (1986) 37.

[34] A. G. Cohen, G. W. Moore, P. C. Nelson, and J. Polchinski, "An Off-Shell Propagator for String Theory," Nucl. Phys. B 267 (1986) 143.

[35] B. S. DeWitt, "Quantum Theory of Gravity 2. The Manifestly Covariant Theory," Phys. Rev. 160 (1967) 1195. 